FNAL TM-2688-APC

ॠF Fermilab CERN-ACC-2018-0036

Revised November 2019

November 21, 2019

alexahin@fnal.gov

frank.schmidt@cern.ch

\title{
Report
}

\section{New SC Algorithm for MAD-X}

\section{Y. Alexahin *}

F. Schmidt ${ }^{\dagger}$

This manuscript has been authored by Fermi Research Alliance, LLC under Contract No. DE-AC02-07CH11359 with the U.S. Department of Energy, Office of Science, Office of High Energy Physics. 


\begin{abstract}
Abstract

MAD-X [1] allows to simulate space-charge effects by approximating the macroparticle ensemble with a Gaussian distribution and using analytic formulas for calculation of the space-charge kick at locations which should be chosen in advance e.g. with V. Kapin's external code [2]. The new MAD-X version features two innovations: a symplectic three-dimensional space-charge kick and Gaussian fit of particle distribution which - in principle - does not require a linearly stable closed optics to exist thus permitting simulations studies of the parametric resonance stopband crossing. It also allows for non stationary distribution so that envelope resonances can be simulated.
\end{abstract}

Keywords: MAD-X, General Accelerator Theory, Beam Optics 


\section{Contents}

$1 \quad$ Symplectic 3D Space-Charge Kick $\ldots \ldots \ldots \ldots \ldots \ldots \ldots$

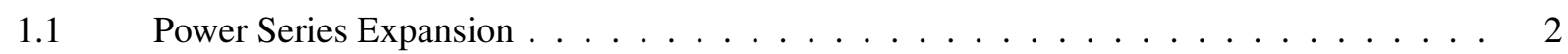

$1.2 \quad$ Asymptotic expansion for halo particles $\ldots \ldots \ldots \ldots \ldots \ldots$

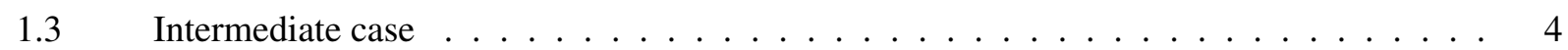

$2 \quad$ Gaussian Fit of Particle Distribution $\ldots \ldots \ldots \ldots \ldots \ldots$

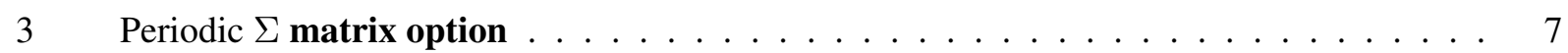

$3.1 \quad$ Algorithm for constructing periodic $\Sigma$ matrix $\ldots \ldots \ldots \ldots \ldots \ldots$

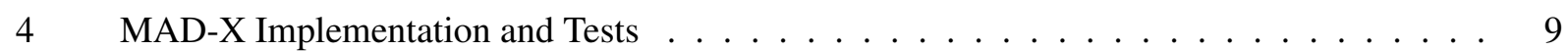

$4.1 \quad$ Implementation of the 3D SC Kick $\ldots \ldots \ldots \ldots \ldots \ldots \ldots$

$4.2 \quad$ CERN PS as a Testcase $\ldots \ldots \ldots \ldots \ldots \ldots \ldots \ldots \ldots \ldots$

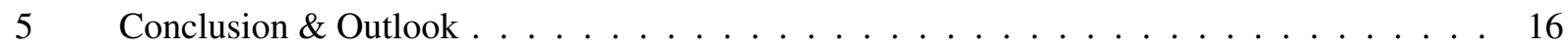

6 Acknowledgments . . . . . . . . . . . . . . . . . . . . . . . . 17

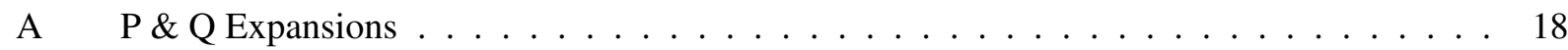

\section{Symplectic 3D Space-Charge Kick}

The kick will be automatically symplectic if it is derived from a potential. We assume the bunch charge density to be of the form

$$
\rho(x, y, z, t)=\frac{\lambda\left(z-v_{0} t\right)}{2 \pi \sigma_{x} \sigma_{y}} \exp \left(-\frac{x^{2}}{2 \sigma_{x}^{2}}-\frac{y^{2}}{2 \sigma_{y}^{2}}\right)
$$

with $\lambda$ being linear charge density. It should not be necessarily Gaussian but the present implementation is limited to a Gaussian profile.

For a long bunch, $\sigma_{z} \gg \max \left(\sigma_{x}, \sigma_{y}\right)$, the space charge potential can be factorized in a similar fashion

$$
\phi(x, y, z, t) \cong \lambda\left(z-\nu_{0} t\right) \cdot \Phi(x, y),
$$

where the two-dimensional potential function $\Phi$ can be presented in the form $[3]^{1}$

$$
\Phi(x, y)=\int_{0}^{1}\left\{\exp \left(-\frac{x^{2} t}{2 \sigma_{x}^{2}}-\frac{y^{2} r^{2} t}{2 \sigma_{y}^{2}\left[1+\left(r^{2}-1\right) t\right]}\right)-1\right\} \frac{\mathrm{dt}}{t \sqrt{1+\left(r^{2}-1\right) t}},
$$

with $r=\frac{\sigma_{y}}{\sigma_{x}}$ for $\sigma_{y}<\sigma_{x}$. If $\sigma_{y}>\sigma_{x}$ one can use formulas with interchanged $x$ and $y$. Computing all components of a quasi-stationary Maxwellian field from the same potential ensures the symplecticity of the associated kick.

Potential function Eq. 3 is regularized and satisfies boundary condition

$$
\left.\Phi(x, y)\right|_{x=y=0}=0 .
$$

As a future development it can be complemented with a longitudinal wake which is independent of the transverse position (in order not to break the symplecticity).

Note [3] discusses the detail of Eq. 3 derivation and considers methods of its numerical calculation in cases of small, large and intermediate values of the transverse displacement in units of beam sizes. Report [4] presents precision tests in these cases. For convenience we cite the major results below.

\footnotetext{
${ }^{1}$ Gaussian units are used. To convert to SI units the r.h.s. should be divided by $4 \pi \varepsilon_{0}$
} 

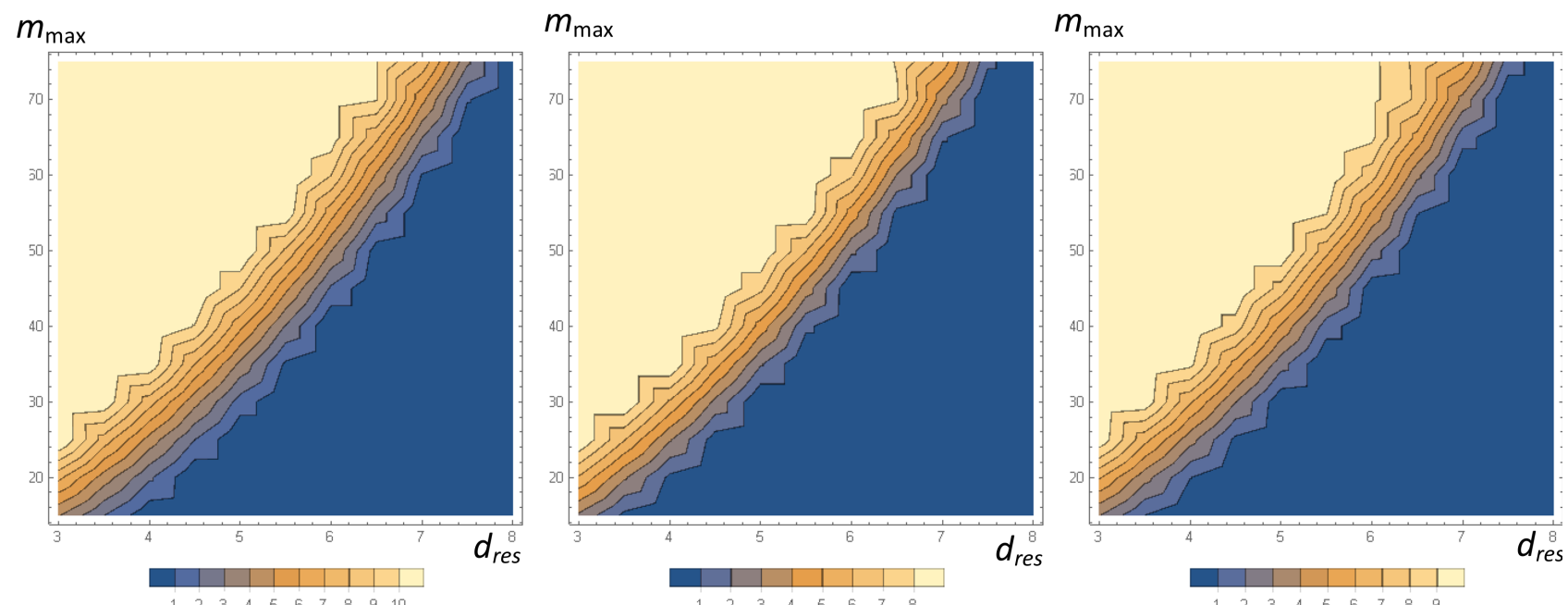

Figure 1: Number of digits of precision Eq. 9 in power series for $\Phi, E_{x}$ and $E_{y}$ as function of displacement Eq. 5 and the highest order $m_{\max }$ of coefficient $B$ used.

\subsection{Power Series Expansion}

For small transverse displacements normalized to respective sigmas

$$
d_{\text {res }}=\sqrt{\frac{x^{2}}{\sigma_{x}^{2}}+\frac{y^{2}}{\sigma_{y}^{2}}} \leq 6
$$

a power expansion can be used:

$$
\Phi=\sum_{m=0}^{M} \sum_{l=0}^{L}\left(-\frac{1}{2}\right)^{m+l}\left(\frac{x}{\sigma_{x}}\right)^{2 m}\left(\frac{\mathrm{ry}}{\sigma_{y}}\right)^{2 l} \frac{1-\delta_{m+l, 0}}{m ! l !} B(m+l-1, l ; a),
$$

where $a=r^{2}-1$ and $\delta_{m, n}$ is the Kronecker delta: $\delta_{m, n}=1$ if $m=n$ and $\delta_{m, n}=0$ otherwise.

Coefficients $B$ are integrals which can be expressed via the Gauss hypergeometric series:

$$
B(m, l ; a) \equiv \int_{0}^{1}\left(\frac{t^{m} \mathrm{dt}}{(1+\mathrm{at})^{l+\frac{1}{2}}}\right)=\frac{1}{m+1}{ }_{2} F_{1}\left(m+1, l+\frac{1}{2} ; m+2 ;-a\right) .
$$

In practice just one integral must be computed, namely the one with $m=m_{\max }, l=0$ (when $|a|<1$ ). Other coefficients are found recursively:

$$
\begin{gathered}
B(m, l ; a)=\frac{1}{m+1}\left[\frac{1}{(1+a)^{l-\frac{1}{2}}}-a\left(m-l+\frac{3}{2}\right) B(m+1, l ; a)\right], \\
B(m, l+1 ; a)=\frac{1}{l+\frac{1}{2}}\left[\frac{1}{(1+a)^{l+\frac{1}{2}}}-\left(m-l+\frac{1}{2}\right) B(m, l ; a)\right] .
\end{gathered}
$$

The Coefficients of Eqs. 7 and 8 determine not only the potential but also the electric field components, so that all necessary quantities can be obtained simultaneously.

The quality of approximation was determined as 


$$
\begin{aligned}
N_{\text {prec }}(\Phi) & =-\log _{10}\left[\frac{|\delta \Phi|}{\Phi_{\max }\left(d_{\text {res }}\right)}\right], \\
N_{\text {prec }}\left(E_{x, y}\right) & =-\log _{10}\left[\frac{\left|\delta E_{x, y}\right|}{\left|\vec{E}\left(d_{\text {res }}\right)\right|_{\text {max }}}\right],
\end{aligned}
$$

with the observation point $(x, y)$ running the ellipse with given $d_{\text {res }}$.

According to Fig. 1 1 with $m_{\max }=75$ more than 6 digits of precision achieved in the potential and the field for up to $d_{\text {res }}=7$.

It should be emphasized that these calculations should be done once for each SC element with particular $a$, but not for each particle.

\subsection{Asymptotic expansion for halo particles}

In the case of large transverse displacements normalized to maximal sigma

$$
d_{\max }=\frac{\sqrt{x^{2}+y^{2}}}{\sigma_{\max }} \geq 6, \sigma_{\max }=\max \left(\sigma_{x}, \sigma_{y}\right),
$$

introducing vectors $\vec{r}$ in the $(x, y)$ plane for brevity, we can write for the Green function satisfying the boundary condition Eq. 4

$$
\begin{aligned}
G^{(1)} & =-\ln \left(\vec{r}-\vec{r}^{\prime}\right)^{2}=-\ln \vec{r}^{2}-\ln \left(1-2 \frac{\vec{r} \cdot \vec{r}^{\prime}}{\vec{r}^{2}}+\frac{\vec{r}^{\prime 2}}{\vec{r}^{2}}\right) \\
& =-\ln \vec{r}^{2}+2 \frac{\vec{r} \cdot \vec{r}^{\prime}}{\vec{r}^{2}}-\frac{\vec{r}^{2} \cdot \vec{r}^{\prime 2}-2\left(\vec{r} \cdot \vec{r}^{\prime}\right)^{2}}{\vec{r}^{4}}+\cdots \\
G^{(t o t a l)} & =G^{(1)}+\ln \vec{r}^{\prime 2},
\end{aligned}
$$
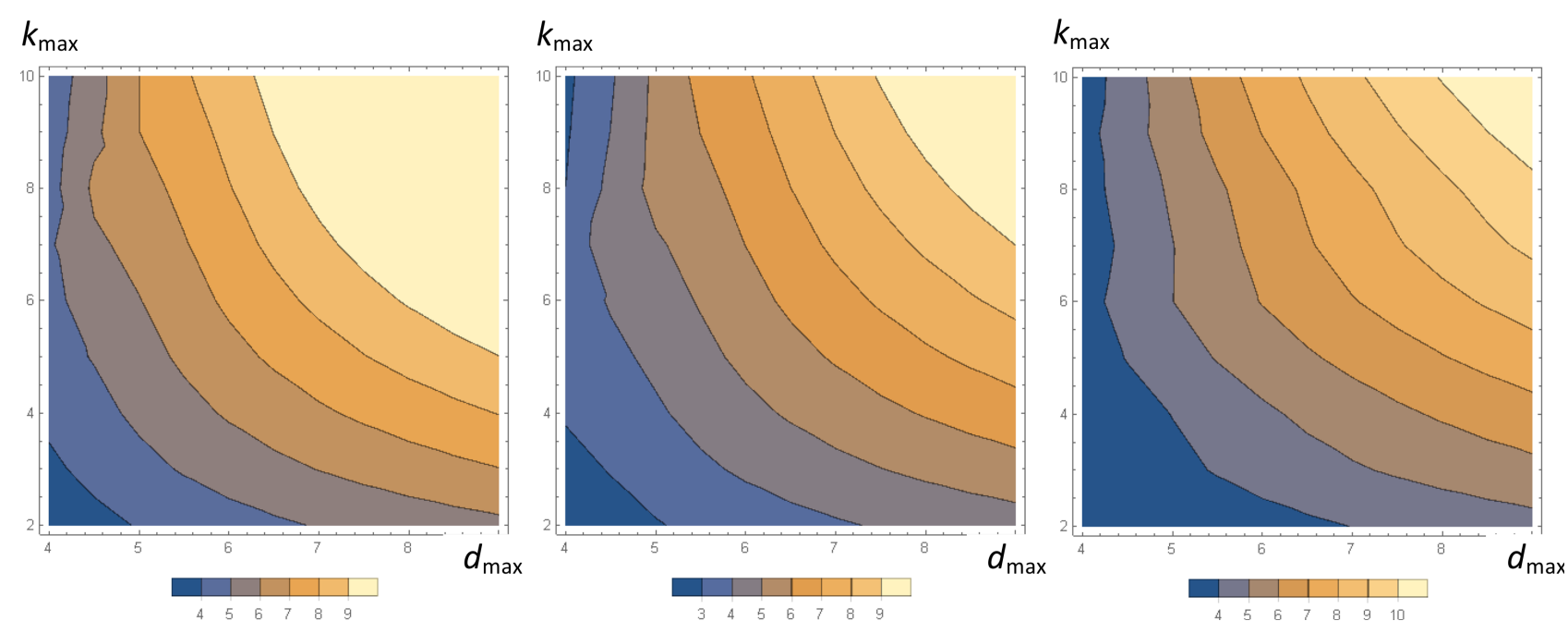

Figure 2: Number of digits of precision in asymptotic expansion of $\Phi, E_{x}$ and $E_{y}$ as function of displacement Eq. 10 and number of terms used.

The contribution to $\Phi$ from the added term evaluates analytically:

$$
\frac{1}{2 \pi \sigma_{x} \sigma_{y}} \iint \ln \left(x^{\prime 2}+y^{\prime 2}\right) \exp \left(-\frac{x^{\prime 2}}{2 \sigma_{x}^{2}}-\frac{y^{\prime 2}}{2 \sigma_{y}^{2}}\right) d x^{\prime} d y^{\prime}=\ln \frac{\left(\sigma_{x}+\sigma_{y}\right)^{2}}{2}-\gamma,
$$


Report

with $\gamma=0.577216 \ldots$ being the Euler gamma.

The total potential function and its derivative can be presented as

$$
\begin{gathered}
\Phi(x, y)=\ln \left[\frac{\left(\sigma_{x}+\sigma_{y}\right)^{2}}{2\left(x^{2}+y^{2}\right)}\right]-\gamma-\frac{1}{\pi} \sum_{k=1}^{k_{\max }} P_{k}(x, y ; r) \frac{\sigma_{x}^{2 k}}{\left(x^{2}+y^{2}\right)^{2 k}}, \\
-\frac{\partial \Phi(x, y)}{\partial x}=\frac{2 x}{x^{2}+y^{2}}+\frac{1}{\pi} \sum_{k=1}^{k_{\max }} Q_{k}(x, y ; r) \frac{\sigma_{x}^{2 k}}{\left(x^{2}+y^{2}\right)^{2 k+1}},
\end{gathered}
$$

where $r=\frac{\sigma_{y}}{\sigma_{x}}$ is assumed. A few first functions $P_{k}$ and $Q_{k}$ computed with the help of Mathematica are given in the Appendix A. The $y$-derivative can be obtained from the above formula by interchange $x \leftrightarrow$ $y$ and $r \leftrightarrow \frac{1}{r}$.

The precision of the asymptotic expansion is shown in Fig. 2. With $k_{\max }=10$ more than 6 digits of precision is achieved for $d_{\max }>6$.

\subsection{Intermediate case}

With large beam ellipse eccentricity, it is possible that $d_{\max }$ for a particle displaced in the direction of smaller beam size is not large enough to make the asymptotic expansion of the previous section work, while $d_{\text {res }}$ is already too large for the power series to convergence as illustrated in Fig. 3 . The larger deviation of the aspect ratio $r$ from 1 the wider the gap left white in Fig. 3

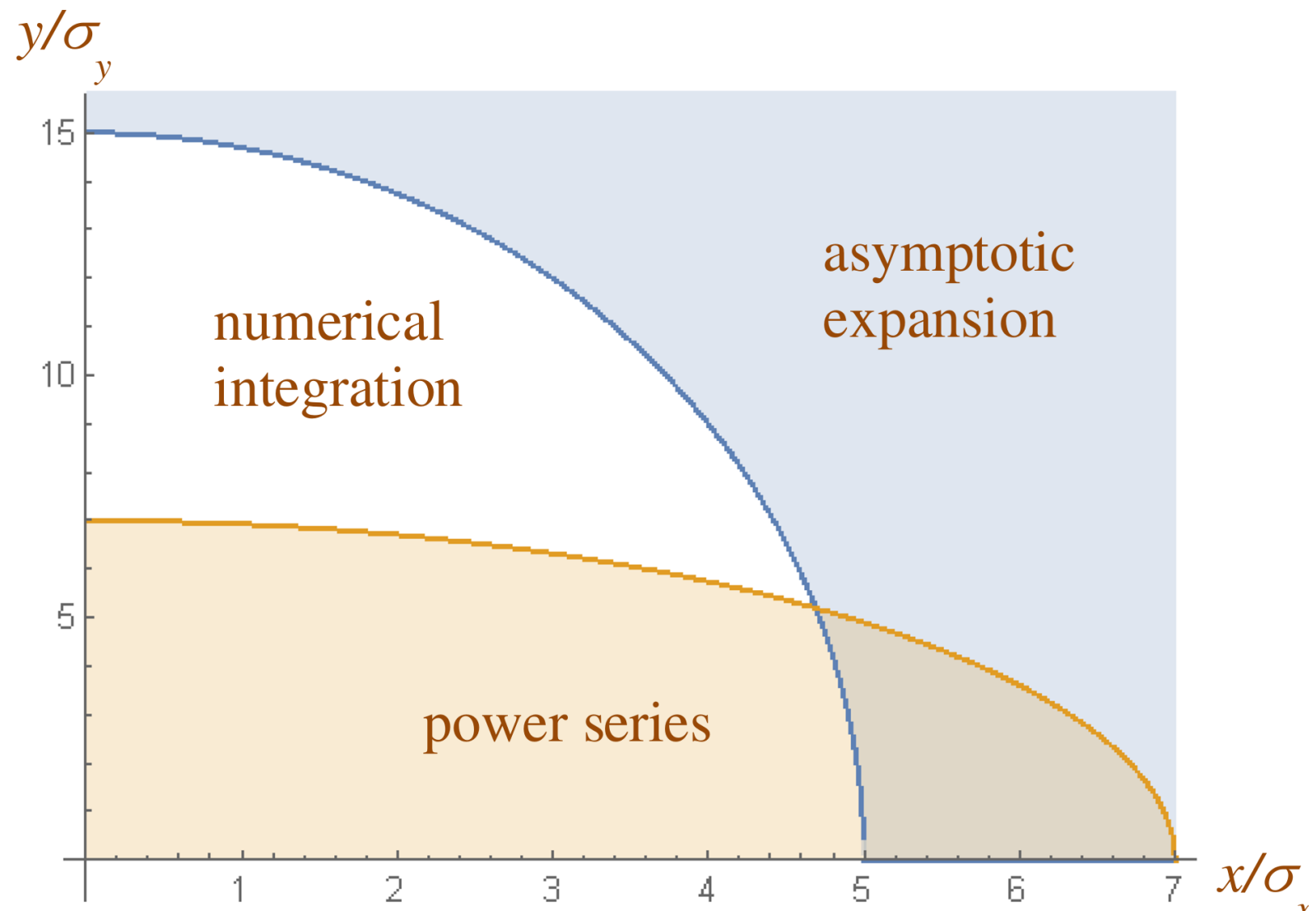

Figure 3: Regions of good precision for power series and asymptotic expansion for aspect ratio $r=\frac{\sigma_{y}}{\sigma_{x}}=\frac{1}{3}$. For coordinates in the white region the numerical integration has to be used.

To calculate the integral in Eq. 3 for particles in this gap let us divide the integration interval in Eq. 3 in two parts: 
Report

$$
\int_{0}^{1} \mathrm{dt}=\int_{0}^{u} \mathrm{dt}+\int_{u}^{1} \mathrm{dt}
$$

with some small $u(u=0.1$ is actually used) and use power series in the first integral:

$$
\int_{0}^{u} \mathrm{dt}=\sum_{m=0}^{M} \sum_{l=0}^{L}\left(-\frac{u}{2}\right)^{m+l}\left(\frac{x}{\sigma_{x}}\right)^{2 m}\left(\frac{y}{\sigma_{y}}\right)^{2 l} \frac{1-\delta_{m+l, 0}}{m ! l !} B(m+l-1, l ; \mathrm{au}) .
$$

The part of the second integral which contains exponential terms can be found by direct numerical integration. The remaining part of the second integral evaluates analytically:

$$
-\int_{u}^{1} \frac{\mathrm{dt}}{t \sqrt{1+\mathrm{at}}}=\left.\log \frac{(1+\sqrt{1+\mathrm{at}})^{2}}{t}\right|_{u} ^{1}
$$

Figure 4 shows the precision of the potential found using the Simpson rule as a function of the number of steps of integration. Errors in $E_{x}$ and $E_{y}$ are about two orders of magnitude lower.

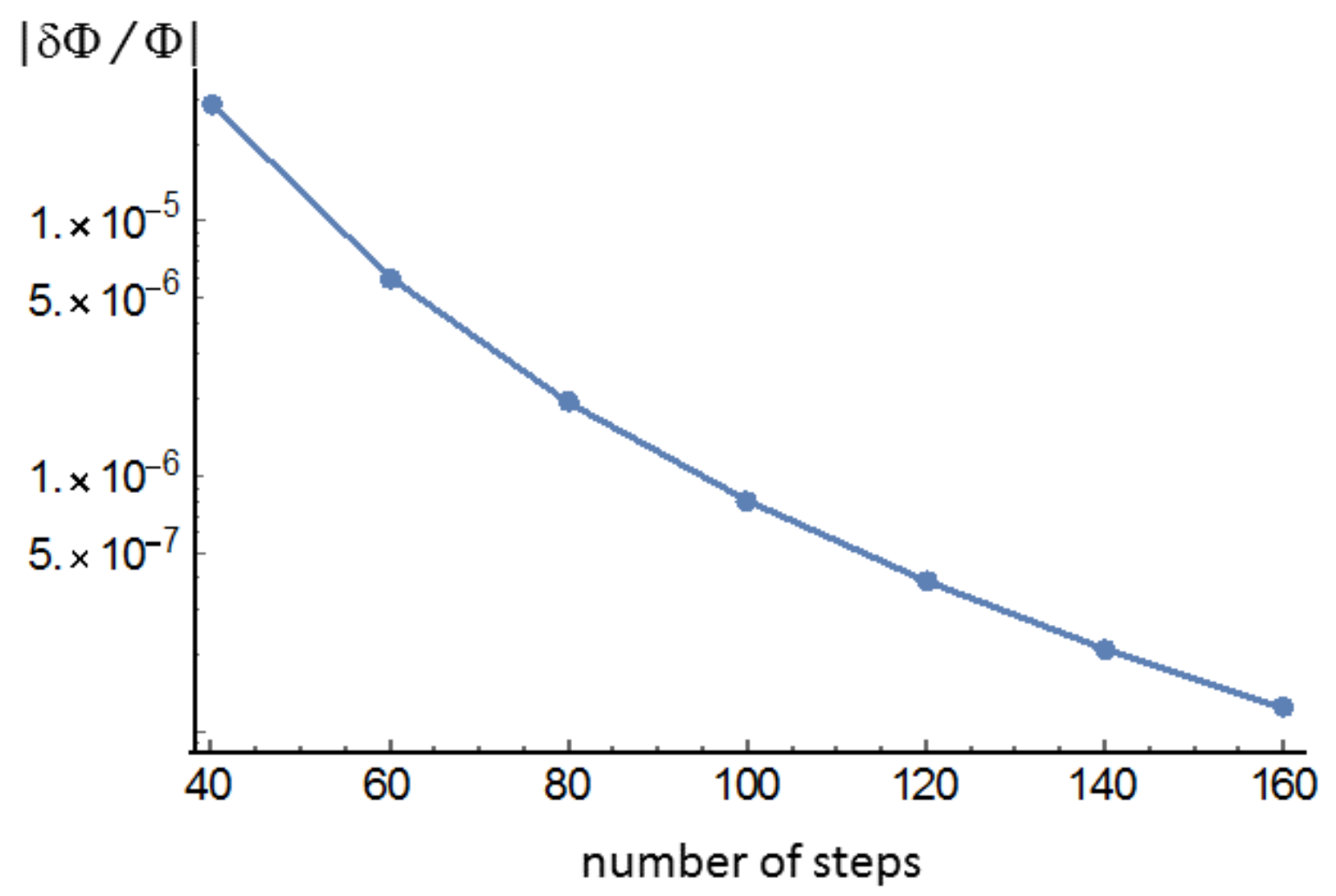

Figure 4: Relative error in $\Phi$ vs. the number of integration steps.

For practical purposes 100 grid points appears to be sufficient.

It should be noted that the expansion coefficients B and weights for numerical integration have to be computed just once for a space-charge element with particular $a$, not for each particle.

\section{Gaussian Fit of Particle Distribution}

The beam sizes should be periodically updated (e.g. every turn) based on evolving particle distribution. The previously implemented algorithm (described a posteriori in [5]) requires stable optics: first to find action variables of the normal modes for each particle at the observation point in order to determine emittances and then to calculate the beam sizes with updated emittances at all space-charge elements. 
The optics functions can also be updated but - even if the stable periodic solution exists - this significantly slows down the computation.

Another drawback of the old algorithm is rather soft suppression of the halo particles contribution to the emittance leading to larger beam sizes and weaker space-charge kicks.

A different approach was proposed in [6] which involves the exact Gaussian fit of particle distribution in terms of the beam $\Sigma$ matrix and its subsequent propagation from the observation point along the lattice using the transfer matrix $\mathrm{T}$ as

$$
\Sigma^{(2)}=T \cdot \Sigma^{(1)} \cdot T^{t}
$$

where superscript " $\mathrm{t}$ " means transposition.

The precision of the fit was checked in [6] only for one-dimensional distribution. Tests with multidimensional distribution revealed problems with spurious coupling which only slowly decreases with the number of particles and in the case of equal emittances does not decrease at all.

The probable cause of the spurious coupling is very strong suppression of contribution of particles with even moderate amplitudes effectively reducing the number of particles. To alleviate this problem the fitting algorithm was modified in the way described below.

First of all let us show that fitting formulas of [6] can be obtained from simple considerations valid for other types of model distribution function as well. Let $z_{i}, i=1, \ldots, n$ be the $n$-dimensional phase space coordinates and $W\left(z_{1}, \ldots, z_{n}\right)$ some weight function that we will use for construction of the $\Sigma$ matrix from a sample of $N$ particles, while trying to approximate this sample with model distribution function $F\left(z_{1}, \ldots, z_{n}\right)$. Below by $z$ we will denote the $n$-tuple $\left(z_{1}, \ldots, z_{n}\right)$.

Let $F(z)$ be the actual normalized beam distribution function. Then - as a consequence of the law of large numbers - we will have for any function $H(z)$ in the limit of large number of particles $N \rightarrow \infty$

$$
\frac{1}{N} \sum_{k=1}^{N} H\left(z^{(k)}\right) \underset{N \rightarrow \infty}{\longrightarrow} \int_{\Omega} H(z) F(z) d^{n} z,
$$

with $\Omega$ being the available phase space.

Now we can define fitted $\Sigma$ matrix using weight function $W(z)$ as

$$
\sum_{i j}^{(f i t)}=\frac{\frac{1}{N} \sum_{k=1}^{N} z_{i}^{(k)} z_{j}^{(k)} W\left(z^{(k)}\right)}{\frac{1}{N} \sum_{k=1}^{N} W\left(z^{(k)}\right)-p},
$$

with $p$ being some parameter. Imposing the requirement that matrix Eq. 20 coincided with the actual $\Sigma$ matrix in the limit $N \rightarrow \infty$

$$
\sum_{i j}^{(e x a c t)}=\int_{\Omega} z_{i} z_{j} F(z) d^{n} z
$$

we have for non-zero elements $\Sigma_{i j}$ (exact)

$$
p=\int_{\Omega} W(z) F(z) d^{n} z-\frac{1}{\sum_{i j}^{(e x a c t)}} \int_{\Omega} z_{i} z_{j} W(z) F(z) d^{n} z .
$$

In the case of Gaussian distribution and weight functions

$$
\begin{aligned}
F(\underline{z}) & =\frac{1}{(2 \pi)^{\frac{n}{2}} \sqrt{\operatorname{det} \sum}} \exp \left[-\frac{1}{2}\left(\underline{\zeta}, \Sigma^{-1} \underline{\zeta}\right)\right], \\
W(\underline{z}) & =\exp \left[-\alpha\left(\underline{\zeta}, \Sigma^{-1} \underline{\zeta}\right)\right],
\end{aligned}
$$

where $\zeta$ are $n$-dimensional vectors of displacements from some average values, we obtain 


$$
p=\frac{2 \alpha}{(1+2 \alpha)^{\frac{n}{2}+1}}
$$

with $\alpha=0, p=0$ and Eq. 20 provides simple r.m.s. values while with $\alpha=\frac{1}{2}, p=\frac{1}{2^{\frac{n}{2}+1}}$ we retrieve the result of [6]. By adjusting $\alpha$ we can suppress the contribution of halo particles while not cutting into the beam core. The choice $\alpha=\frac{1}{4}$ greatly reduced the spurious coupling. It should be noted that with $\alpha \neq \frac{1}{2}$ this fit does not render the minimum of the integrated squared difference in the phase density of actual particle distribution and the model distribution and in this sense is not exact. However, it leads to more practical results.

Since the $\Sigma$ matrix is not known, Eq. 20 is a non-linear equation which can be solved iteratively. The full set of equations to solve is:

$$
\begin{gathered}
\underline{a}=\frac{\sum_{k=1}^{N} \underline{z}^{(k)} \exp \left[-\alpha\left(\underline{\zeta}^{(k)}, \Sigma^{-1} \underline{\zeta}^{(k)}\right)\right]}{\sum_{k=1}^{N} \exp \left[-\alpha\left(\underline{\zeta}^{(k)}, \Sigma^{-1} \underline{\zeta}^{(k)}\right)\right]}, \underline{\zeta}^{(k)}=\underline{z}^{(k)}-\underline{a}, \\
\sum_{i j}=\frac{\frac{1}{N} \sum_{k=1}^{N} \zeta_{i}^{(k)} \zeta_{j}^{(k)} \exp \left[-\alpha\left(\underline{\zeta}^{(k)}, \Sigma^{-1} \underline{\zeta}^{(k)}\right)\right]}{\frac{1}{N} \sum_{k=1}^{N} \exp \left[-\alpha\left(\underline{\zeta}^{(k)}, \Sigma^{-1} \underline{\zeta}^{(k)}\right)\right]-\frac{2 \alpha}{(1+2 \alpha)^{\frac{n}{2}+1}}},
\end{gathered}
$$

The iterative procedure for Eqs. 25 and 26 has a tendency to overshoot, the convergence can be improved by introduction of a damping factor $0<d<1$ :

$$
\Sigma_{l}=d \Sigma_{l}^{(\text {formula })}+(1-d) \Sigma_{l-1},
$$

where $l$ is the iteration number. Tests showed that $d=0.85$ is close to the optimum.

\section{Periodic $\Sigma$ matrix option}

The $\Sigma$ matrix propagated along the lattice according to Eq. 18 may contain a free-oscillating part due to e.g. initial mismatch. This gives us the ability to study the effects of interaction of individual particles with coherent (envelope) oscillations, such as halo formation [7] and Landau damping.

With a finite number of macro-particles there are inevitable statistical fluctuations which act as a never stopping pump increasing beam emittance through this interaction. The obvious remedy illustrated by Fig. 5- a larger number of macro-particles - defeats our goal of the speed of simulations.

Thus we have to consider two modes of operations: freely propagating $\Sigma$ and periodic $\Sigma$. The first option allows to study envelope resonances but requires maximum available number of particles to suppress the statistical noise. The second option is analogous to the existing MAD-X algorithm but more efficiently suppresses the halo contribution to emittances and includes effects of the machine coupling (we plan to add coupling through the space charge forces in the future).

The periodic $\Sigma$ option requires the stable optics to exist and involves computation of the eigen-mode emittances $\varepsilon_{m}, m=1,2,3$, which are imaginary parts of eigenvalues of matrix

$$
\Omega=\Sigma S,
$$

with $S$ being the symplectic unity matrix

$$
S=\left(\begin{array}{cc}
0 & 1 \\
-1 & 0
\end{array}\right) \oplus\left(\begin{array}{cc}
0 & 1 \\
-1 & 0
\end{array}\right) \oplus\left(\begin{array}{cc}
0 & 1 \\
-1 & 0
\end{array}\right)
$$




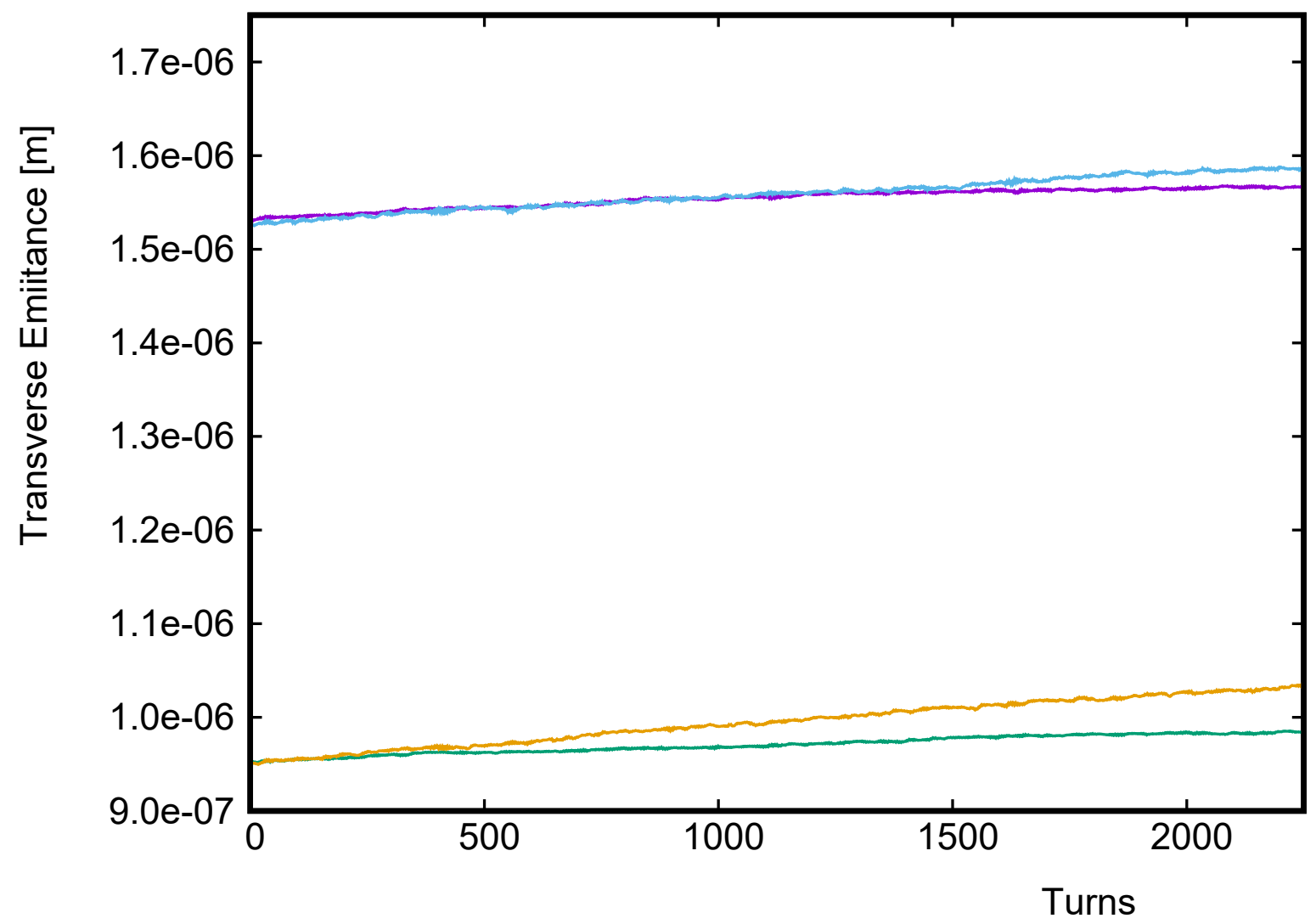

Figure 5: Vertical and horizontal emittance growth (two lower and two upper curves respectively) of the PS in the freely propagating $\Sigma$ mode: blue and yellow curves - for 8000 macro-particles, violet and cyan - for 16000 macro-particles.

\subsection{Algorithm for constructing periodic $\Sigma$ matrix}

After completing turn $n-1$, we obtain the transfer matrix $\mathrm{T}(n-1)$ and the particle distribution at the starting point of turn $n$. Using the algorithm of the previous Section we calculate $\Sigma(n)$ but instead of propagating it around machine we use it for computing normal mode emittances $\varepsilon_{m}$ from matrix Eq. 28, To build a (quasi)periodic $\widetilde{\Sigma}(n)$ at the starting point of turn $n$ we also need the eigenvectors of the 1-turn transfer matrix that has three complex-conjugate pairs of eigenvalues and eigenvectors

$$
T \underline{\nu}_{k}=\lambda_{k} \underline{\nu}_{k}, \lambda_{2 m}=\lambda_{2 m-1}^{*}, \underline{\nu}_{2 m}=\underline{\nu}_{2 m-1}^{*}
$$

where $m=1,2,3$ is the mode number, asterisk denotes complex conjugation, underscore means $6 \mathrm{D}$ vector. We choose normalization as in MAD

$$
\left(\underline{\nu}_{2 m-1}^{*}, S \underline{\nu}_{2 m-1}\right)=2 i,
$$

that can be rewritten for real and imaginary parts of the eigenvectors as

$$
\begin{aligned}
& \left(\underline{\nu}_{i}^{\prime}, \mathrm{S} \underline{\nu}_{j}^{\prime}\right)=\left(\underline{\nu}_{i}^{\prime \prime}, \mathrm{S} \underline{\nu}_{j}^{\prime \prime}\right)=0, \\
& \left(\underline{\nu}_{2}^{\prime}, \mathrm{S} \underline{\nu}_{2 n-1}^{\prime \prime}\right)=\delta_{m n}, \\
& \underline{\nu}_{i} \equiv \operatorname{Re}\left(\underline{\nu}_{i}\right), \quad \underline{\nu}_{i}^{\prime \prime} \equiv \operatorname{Im}\left(\underline{\nu}_{i}\right) .
\end{aligned}
$$

In assumption that the new transfer matrix, $\mathrm{T}(n)$, will not differ much from $\mathrm{T}(n-1)$, we can use eigenvectors of T $(n-1)$ to build $\widetilde{\Sigma}(n)$ according to [8 10 ]

$$
\widetilde{\Sigma}_{i k}=\sum_{m=1}^{3} \epsilon_{m}\left(\nu_{2 m-1, i}^{\prime} \nu_{2 m-1, k}^{\prime}+\nu_{2 m-1, i}^{\prime \prime} \nu_{2 m-1, k}^{\prime \prime}\right),
$$


where $\nu_{m, k}$ means $k$-th component of $m$-th eigenvector. The eigenvectors can be propagated around the ring for Eq. 33 to be applied at every space-charge element. Alternatively, $\widetilde{\Sigma}(n)$ can be propagated around the ring using sectormaps.

\section{MAD-X Implementation and Tests}

The first macro based MAD-X SC implementation has been done and reported in 2010 by V. Kapin and Y. Alexahin [2]. During a three month visit to Fermilab Frank, in close collaboration with V. Kapin, managed to include a large fraction of the SC treatment directly into MAD-X while keeping the SC lattice set-up in separate external steps [11].

During 2013 and 2014 at BNL [12] MAD-X has been instrumented with OPENMP compiler directives to allow to use several cores in parallel. A speed-up of roughly a factor of 5 has been achieved.

At CERN it was decided to include Frank's SC implementation into the main branch of the MAD$\mathrm{X}$ development [1].

The next step has been to make use of the $\Sigma$ matrix approach in an attempt to determine the lattice TWISS parameters directly from the distribution of the particles rather than having to stop the simulation to perform a MAD-X TWISS calculation. The Mathematica package had been transferred into an efficient FORTRAN implementation. Using the Fermilab Booster, as an example, we experienced an apparently inexplicable artificial coupling between the transverse planes that rendered this technique not applicable for real world examples (but see below).

M. Titze has contributed to the SC implementation in MAD-X by a symplectic treatment of thin combined function magnets [13]. It is true that this had been solved in a general way by E. Forest for thick elements described in his first book that is the base of his PTC [14] code. PTC is intimately linked with MAD-X. However, since we need to start with a thin MAD-X lattice we cannot take advantage of this general solution. In a nutshell, the most simple combined function magnet which is a dipole and quadrupole (E.G. the CERN PS) adds effectively a pseudo sextupole which has a significant effect on the chromaticity of about $20 \%$ for the PS.

There has been a review of the set-up macros and independently it was discovered [15, 16] that the dispersive part of transverse emittance had been ignored.

During a CERN visit by Y. Alexahin, we found that the treatment of the longitudinal plane in the SC adaptive mode had been too aggressive and needed to be removed. The adaptive mode is now restricted to the transverse case only. It was also found that the adaptive SC mode creates numerical noise both locally and globally. Figure 6 shows that for the PS the local noise goes down by roughly a factor of two what might be expected when raising the number of macro-particles from $1 \mathrm{k}$ to $4 \mathrm{k}$, i.e. by a factor of four. The global noise or long-term blow-up is also largely reduced. 


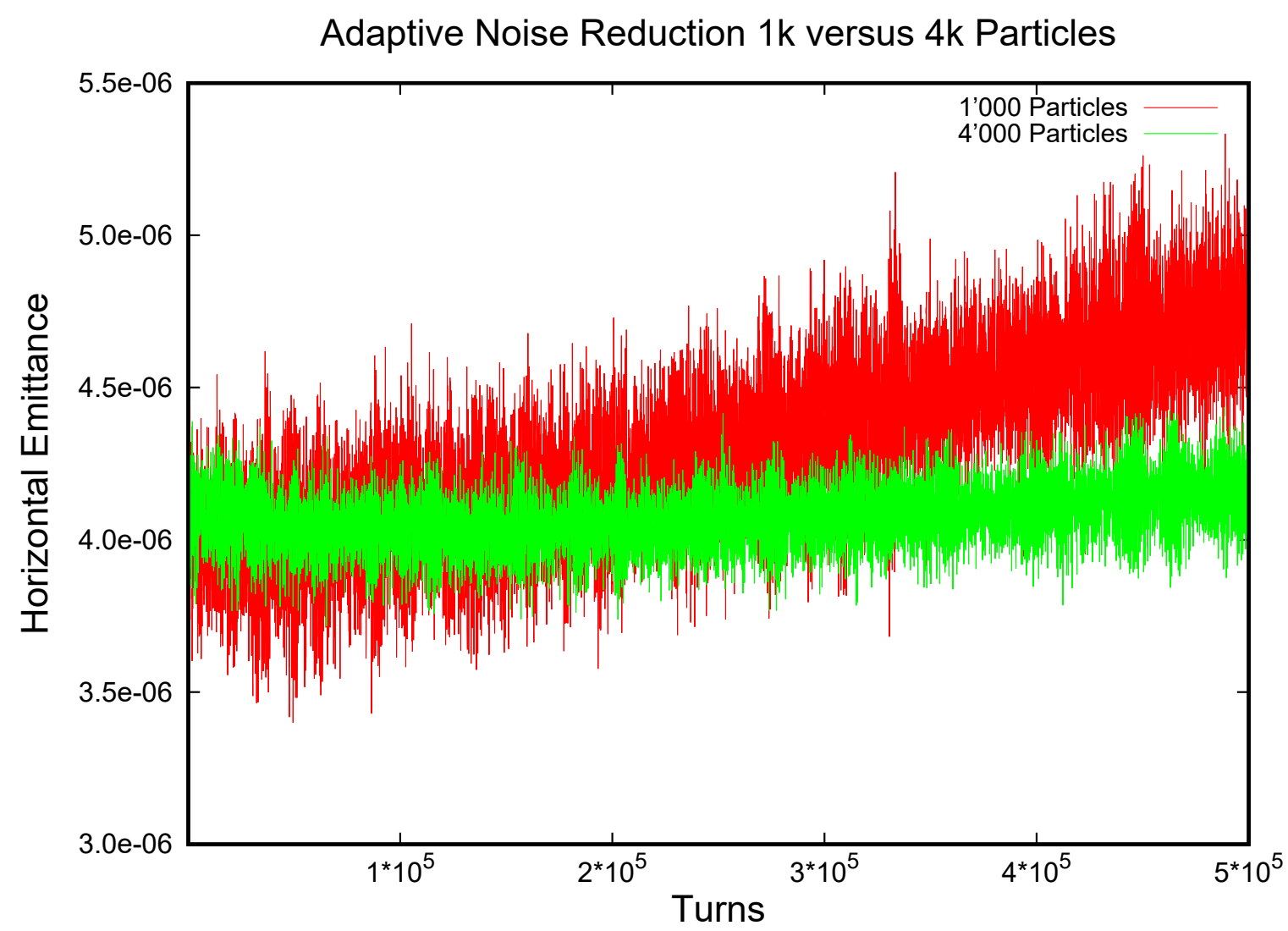

Figure 6: 2017 horizontal emittance evolution versus turn number for the CERN PS for $1 \mathrm{k}$ and $4 \mathrm{k}$ macro-particles respectively. The MAD-X simulations have been done in the SC adaptive mode. Both local and global noise decreases with the number of macro-particles, the former by a factor of 2 .

\subsection{Implementation of the 3D SC Kick}

The initial work on the symplectic 3D SC kick had been started in 2017. To this end the Mathematica implementation of the theory as outlined in Sec1 1 had been transferred to a stand-alone package written in FORTRAN, the same has been done for the Mathematica notebook for the beam $\Sigma$ matrix as described in Sec.2.

The implementation of the FORTRAN packages for the 3D SC Kick and the beam $\Sigma$ matrix treatment into a MAD-X test version was done first.

This new SC mode has become an additional, flag driven, feature while keeping the older adaptive and purely frozen versions intact. The new SC mode comes in two flavors Self-consistent (Sec. 2) or Periodic $\Sigma$ matrix (Sec. 3) each controlled by a flag.

Adding these new features gave us a chance to review carefully what we already had. It turned out that the expected intensity dependence of the SC force had been ignored but this is now fixed. In this context, it was also noted that the purely frozen SC mode actually had a small jitter due to a relic of an early version of the adaptive mode that had to be taken out.

One of the main outcomes has been the discovery that the $\Sigma$ matrix calculation suffers from a singularity when the two transverse emittances are of equal size. This has been the case for the Fermilab Booster (see above). This important result will be published in a separate paper.

In general, the $\Sigma$ matrix reacts very sensitively on any artifact that the beam distribution might exhibit. For example, we have noticed yet another case where the $\Sigma$ matrix did not provide sensical results. On inspection, we found that some of six coordinates in the beam distribution had been created with accidentally correlated random numbers. Such defects of the beam distribution are hardly detected otherwise.

Essential is also a comparison of computational speed of the 3D SC Kick (Periodic $\Sigma$ matrix) with 
the adaptive mode. Long-term simulations for the CERN PS Booster lasted about 10\% longer for the 3D Kick version, which is quite acceptable given the advantages.

The computational speed for the "Self-consistent mode" is another issue since it depends on how much artificial emittance blow-up due to numerical noise can be tolerated. As a consequence this mode is not really applicable for long-term studies. In fact, that is not much different from what is known from PIC codes.

\subsection{CERN PS as a Testcase}

For the long-term simulation test, using the periodic $\Sigma$ matrix option, the CERN PS was chosen with the beam parameter as found in TAB. 1. In particular, we are using the recorded beam profile [17] s of the PS experiment [18].

Simulation results are compared for four working points that have also been studied in the experiment:

1) Far from resonances: Bare Tunes: $Q_{x}=6.244, Q_{y}=6.465$,

2) Close to the $Q_{x}+2 Q_{y}$ resonance: Bare Tunes: $Q_{x}=6.104, Q_{y}=6.476$,

3) SC Tune shift slightly above integer resonance $Q_{x} \sim 6.01$ : Bare Tunes: $Q_{x}=6.060, Q_{y}=6.478$,

4) SC Tune shift on top of integer resonance $Q_{x} \sim 5.989$ : Bare Tunes: $Q_{x}=6.039, Q_{y}=6.479$.

In the following figures $(7)$ through 10$)$ the experimental profiles from the PS wire-scanners are compared with profiles generated from simulation data. For a meaningful comparison one has to realize that a wire-scan is lasting for approximately 1'000 turns in the PS. Therefore, in the simulations we take the $\mathrm{x}$ and $\mathrm{y}$ coordinates at the start from 0 to $1^{\prime} 000$ turns or at the end from $500 \mathrm{k}$ to $501 \mathrm{k}$ turns and for the simulated 2'000 macro-particles. A histogram is taken such that a smooth profile is obtained and then the beta-functions are scaled to the location of the wire-scanners. The vertical scale is adapted so that the experimental and simulation profiles have the same maximum, therefore one finds "arbitrary units" on the vertical axis of the figures. The only other operations is to shift the experimental profiles such that the maximum amplitude is found at zero $\mathrm{x}$ or $\mathrm{y}$ values.

Figure 7 shows that far from resonances (Bare Tunes: $Q_{x}=6.244, Q_{y}=6.465$ ) the simulations reproduce the measured horizontal and vertical profiles very well both at the start and after 500k turns. In Fig. 8 the motion is in the vicinity of the $Q_{x}+2 Q_{y}$ resonance (Bare Tunes: $Q_{x}=6.104, Q_{y}=6.476$ ). The initial profiles both horizontal and vertical agree well. It is remarkable how well the broadening of horizontal profile has been reproduced in simulations. Even more so in the vertical plane where shoulders are quite well reproduced.

Going close but above the integer (Fig. 9 with bare tunes of $Q_{x}=6.060, Q_{y}=6.478$ the agreement of the start profiles remain good, albeit slightly wider in both planes in the simulations. On top of the integer (Fig. 10 ) with bare tunes of $Q_{x}=6.039, Q_{y}=6.479$ the measured horizontal profile show somewhat more initial blow-up over 1'000 turns, while the vertical profile looks very close indeed. This will require some fine-tuning in the set-up of the simulations. Long-term simulations have not been performed for these last two cases. 
Table 1: CERN PS beam and machine parameters taken from Ref. [18].

\begin{tabular}{lc} 
Parameter & Value \\
\hline Intensity $N_{p}\left[10^{10} \mathrm{p}\right]$ & 55 \\
Normalized horizontal rms emittance $\varepsilon_{x}^{n}[\mathrm{~mm} \mathrm{mrad}]$ & 3.6 \\
Normalized vertical rms emittance $\varepsilon_{y}^{n}[\mathrm{~mm} \mathrm{mrad}]$ & 2.2 \\
Bunch length $\sigma_{t}[\mathrm{~ns}]$ & 33 \\
Momentum spread $\frac{\Delta p}{p}\left[10^{-3}\right]$ & 0.95 \\
Horizontal maximum tune spread $\Delta Q_{x, \max }{ }^{1}$ & -0.05 \\
Vertical maximum tune spread $\Delta Q_{y, \max }{ }^{1}$ & -0.07 \\
Sextupole current $I_{\mathrm{SX}}[\mathrm{A}]$ & 2 \\
Harmonic number $h$ & 8 \\
RF voltage $V_{\mathrm{RF}}[\mathrm{kV}]$ & 20.5 \\
Natural horizontal chromaticity $Q_{x}^{\prime}$ & -5.30 \\
Natural vertical chromaticity $Q_{y}^{\prime}$ & -7.02 \\
Kinetic energy of the stored beam [GeV] & 2 \\
Number of stored turns & 497646 \\
Storage time $[\mathrm{s}]$ & 1.1 \\
Relativistic $\beta$ & 0.948 \\
Relativistic $\gamma$ & 3.14 \\
Synchrotron period [turns] & 1164 \\
$\beta_{x}$ at the horizontal wire scanner in SS68 [m] & 12.40 \\
$\beta_{y}$ at the vertical wire scanner in SS64 [m] & 21.75
\end{tabular}

The testing of the new 3D SC kick method (periodic $\Sigma$ matrix) has been successfully concluded and one finds good agreement with the experimental expectations.

Our plans beyond the results, presently achieved, can be summarized below with four new work units:

- In 2013 the original SC implementation has been instrumented with OPENMP directives [12] with a maximum performance gain of a factor of 5. Similarly, this effort has to be repeated for the new implementation. In fact, it has already been found [20], via code profiling, that the code part with the highest number of floating point operations is located in the routine where the 3D SC kick is calculated. It is interesting to note that the OPENMP feature had been temporarily disabled due to a conflict with another MAD-X module.

- In all SC implementations the implicit assumption is that the SC kick does not couple the transverse motion planes. Following the work by G. Ripken [9] we will make use of the transverse coupling terms of the $\Sigma$ matrix.

- Another limitation is the fact that the beam distributions may not even be close to a Gaussian profile, in particular in the longitudinal plane. The goal is to allow for more general beam shape distributions mainly for the longitudinal plane.

- Last, we will have to address of a better set-up of the initial profiles found in the experiment, in particular at the integer resonance. 
Hor. $Q x=6.244, Q y=6.465$

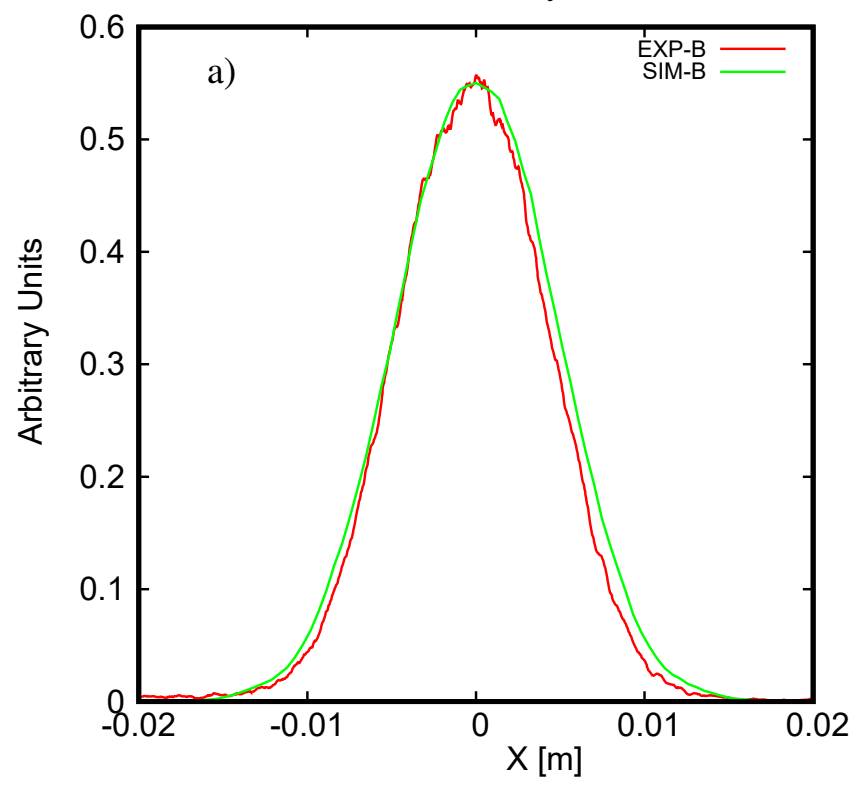

Ver. $Q x=6.244, Q y=6.465$

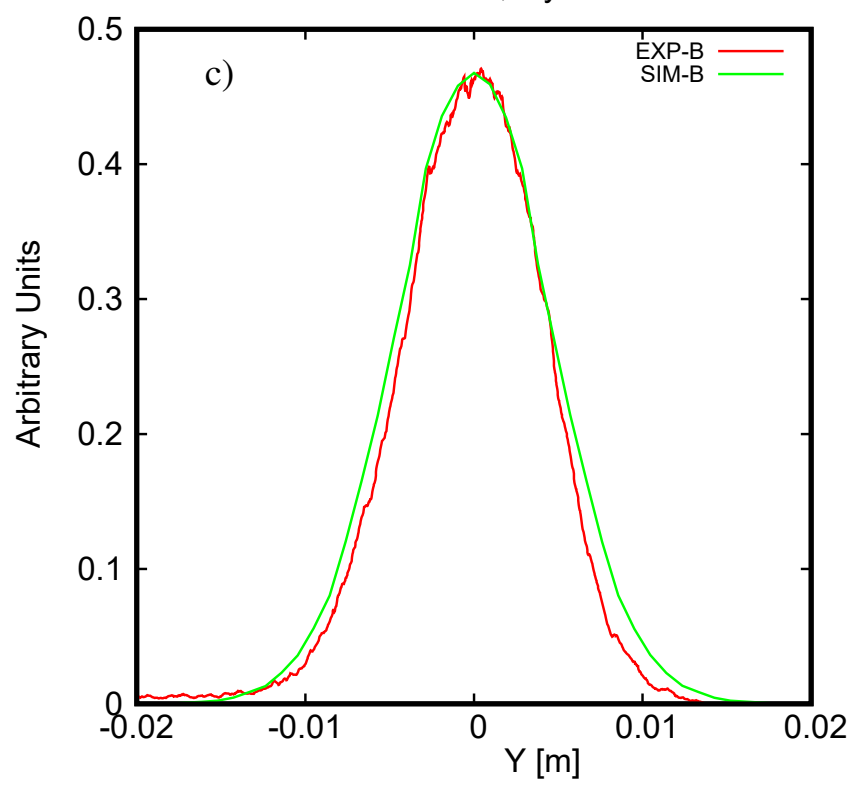

Hor. $Q x=6.244, Q y=6.465$

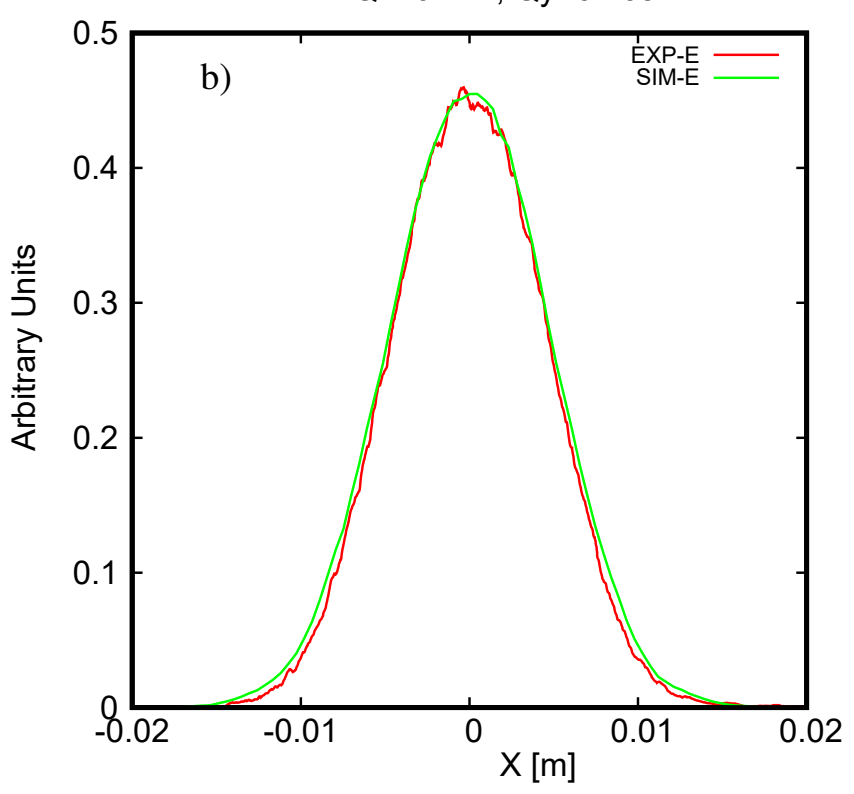

Ver. $Q x=6.244, Q y=6.465$

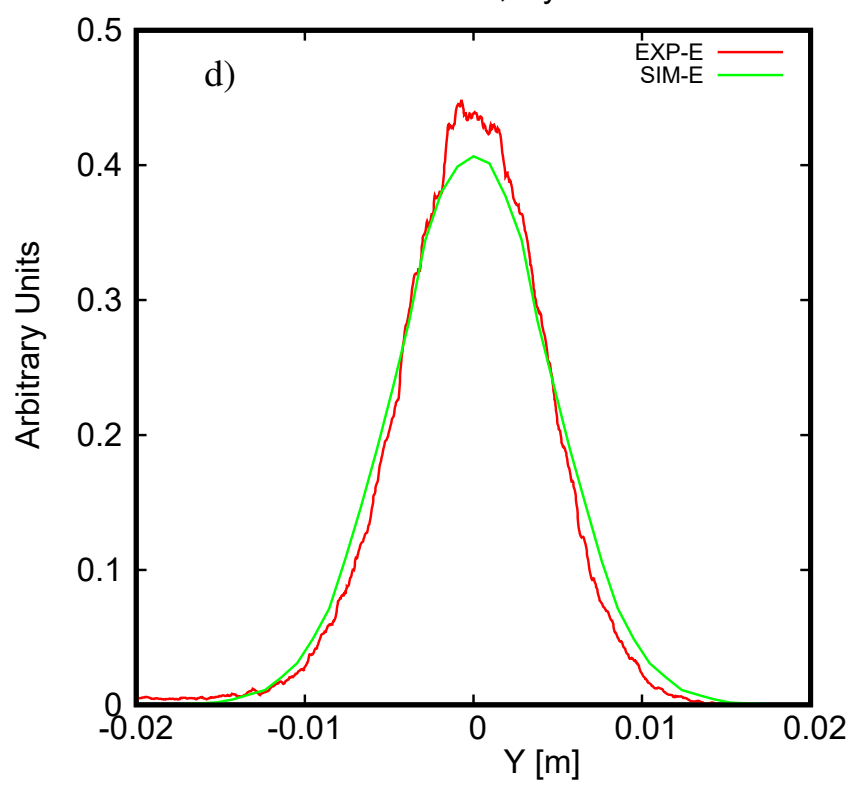

Figure 7: Far from resonances: Bare Tunes: $Q_{x}=6.244, Q_{y}=6.465$; Start profiles on the left and at the end on the right; The transverse beam profiles are in excellent agreement both at the start and end with 0 and 500k turns respectively. 

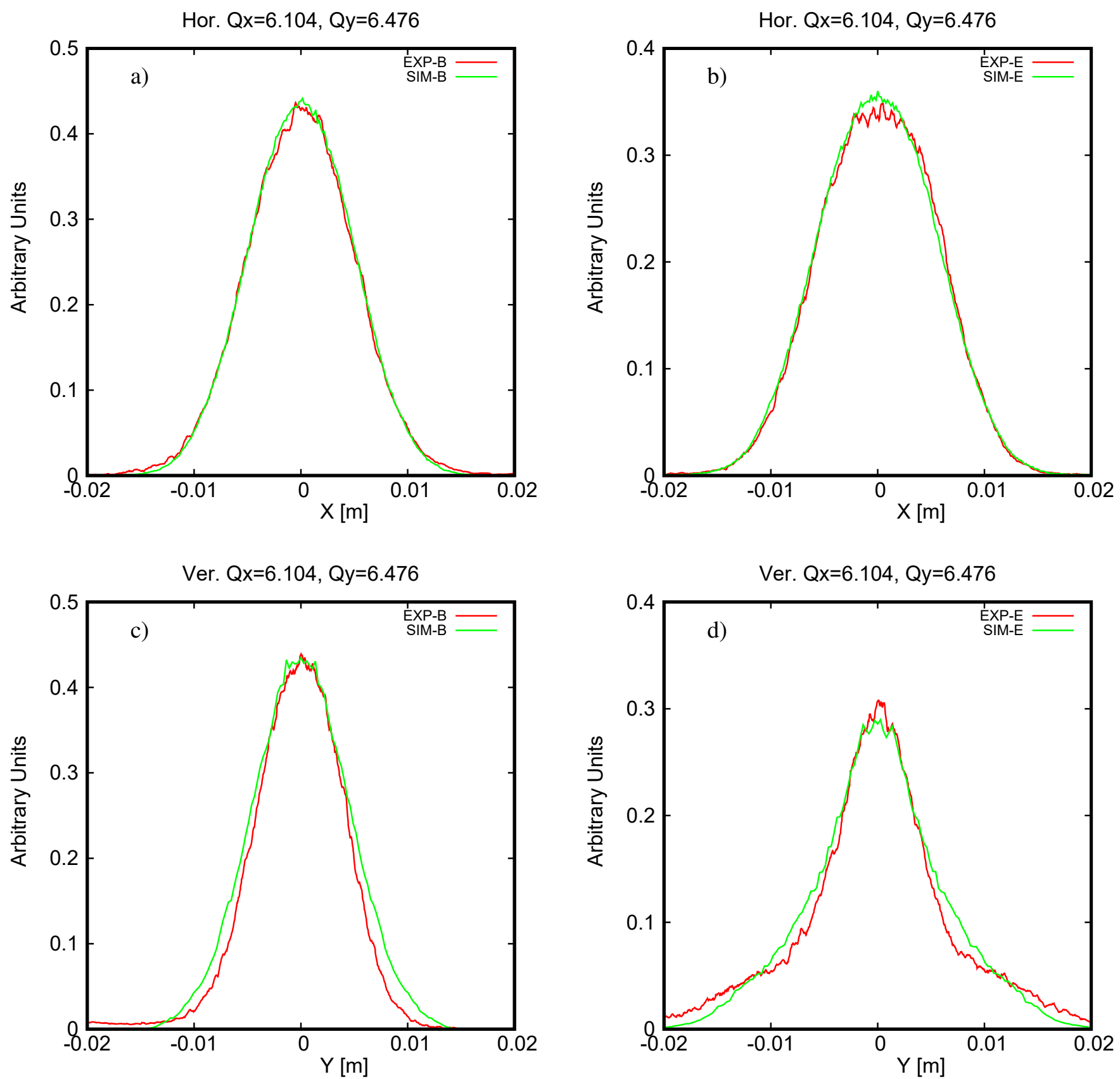

Figure 8: Close to the $Q_{x}+2 Q_{y}$ resonance: Bare Tunes: $Q_{x}=6.104, Q_{y}=6.476$; Start profiles on the left and at the end on the right; The transverse beam profiles are in excellent agreement both at the start and end with 0 and $500 \mathrm{k}$ turns respectively. Notice that the horizontal profiles have widened, while the vertical shoulders have been well reproduced in the simulations. 

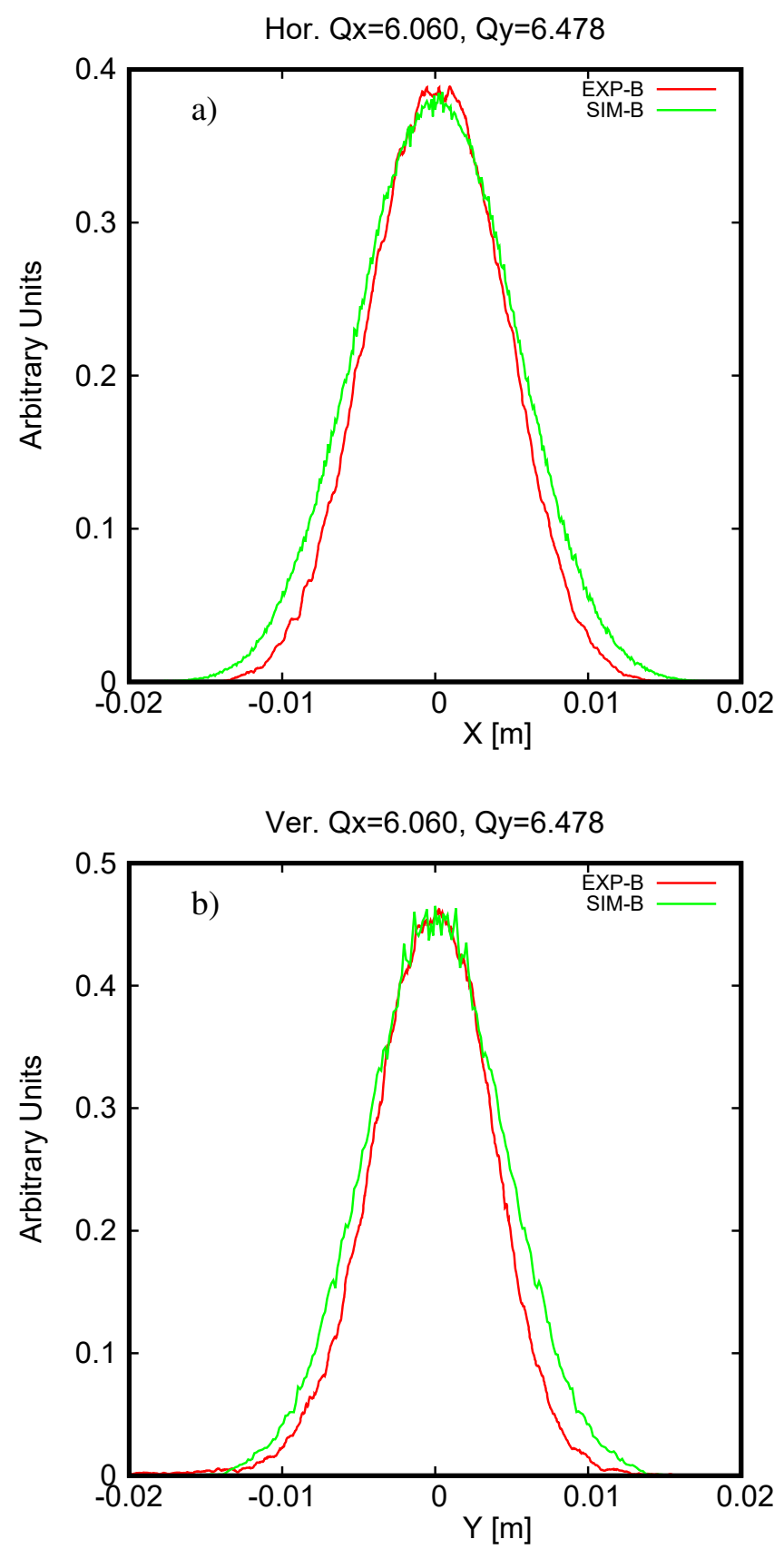

Figure 9: SC Tune shift slightly above integer resonance $Q_{x} \sim 6.01$ : Bare Tunes: $Q_{x}=6.060, Q_{y}=6.478$; In this case only the start profiles are compared. The agreement is good although one can argue that the profiles are slightly wider in the simulations. 
Hor. Qx=6.039, Qy=6.479

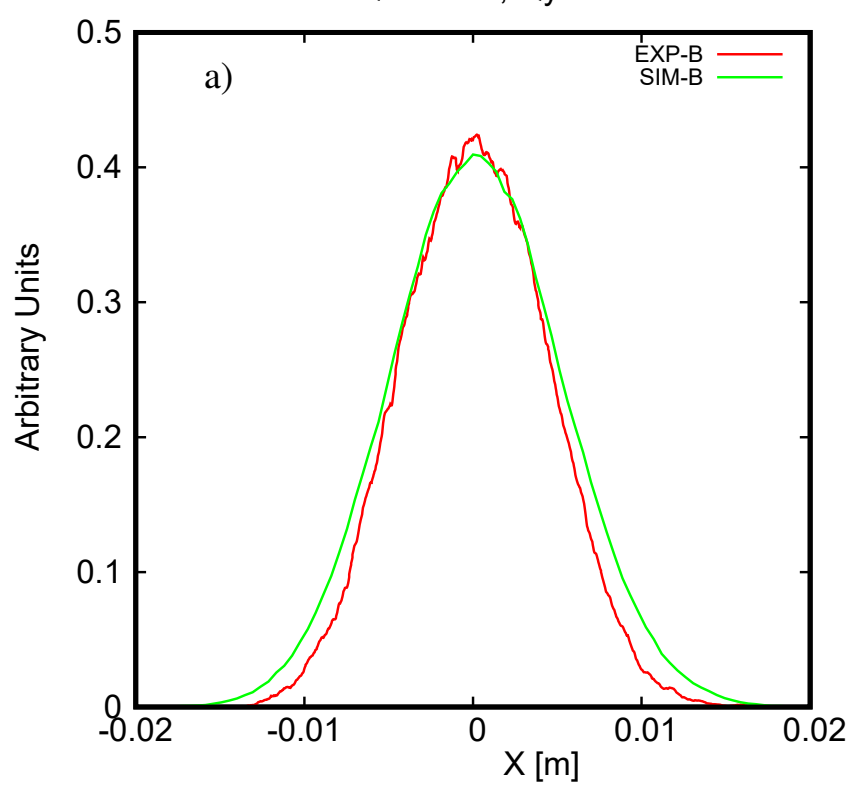

Ver. Qx=6.039, Qy=6.479

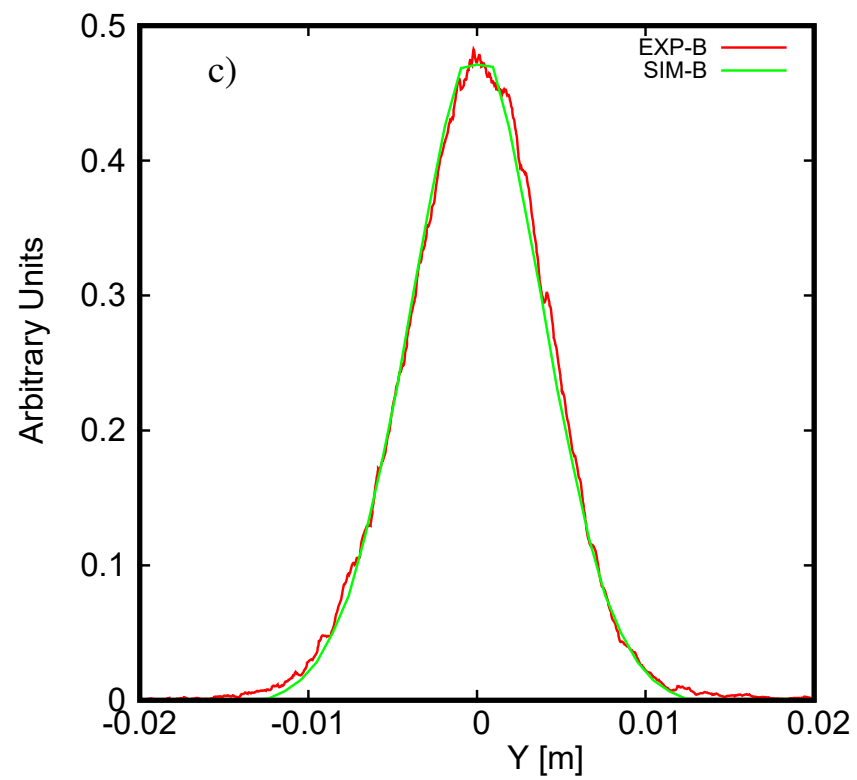

Hor. $Q x=6.039, Q y=6.479$
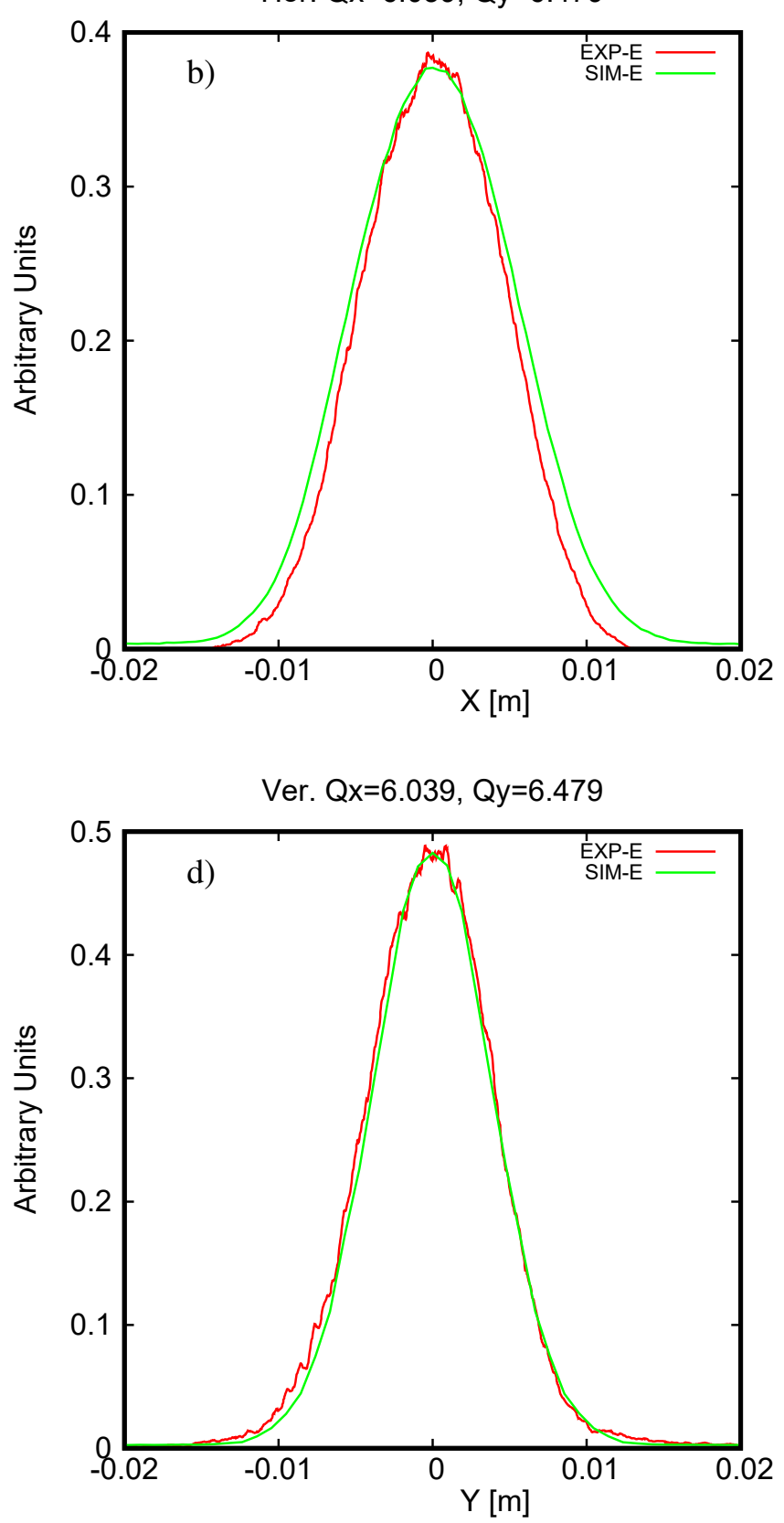

Figure 10: SC Tune shift on top of integer resonance $Q_{x} \sim 5.989$ : Bare Tunes: $Q_{x}=6.039, Q_{y}=6.479$; The vertical profiles from the simulations agree very well with the experimental ones both horizontally and vertically, except that in the horizontal plane the simulations show a wider profiles than in the experiment.

\section{Conclusion \& Outlook}

A new 3D SC symplectic kick has been developed together with the $\Sigma$ matrix treatment to determine the machine optics and the emittance at any moment in the simulations. This is required to determine the strength of the SC force at every turn. This formalism has been implemented into a special test version of MAD-X and has been properly checked for the case of the CERN PS machine.

There are a number of issues and new desirable features that still needs to be added to the present implementation. However, this new method is now matured enough to be made available in the general MAD-X version. In fact, we are expecting that it will also become of use in other codes. 
The adequate setting-up of the simulations needs further care and understanding, in particular at strongly excited resonances like the $1^{\text {st }}$ order or integer resonance.

\section{Acknowledgments}

The authors are grateful to V. Shiltsev and A. Valishev (FNAL APC) for invariable support of this work. It was partially supported by Fermilab, operated by Fermi Research Alliance, LLC under Contract No. DE-AC02-07CH11359 with the United States Department of Energy.

We would like to acknowledge Alex Huschauer for providing the profile data-sets of the 2012 experiment [18]. Malte Titze provided the code and integration of the thin combined function magnet [13] into MAD-X [1]. Both Malte and Alexander Valishev are thanked for a critical review of the SC set-up in MAD-X.

\section{Bibliography}

[1] L. Deniau (editor), H. Grote, G. Roy, F Schmidt, "The MAD-X Program (Methodical Accelerator Design)", Version 5.04.01, User's Reference Manual.

[2] V. Kapin and Y. Alexahin, "Space Charge Simulation Using MADX with Account of Synchrotron Oscillations", Proc. XXII Russian Particle Accelerator Conference RuPAC-2010, Protvino, Moscow region, Oct 27, 2010, pp. 204-206.

[3] Y. Alexahin, FNAL Beams-doc-5032-v3, 2017.

[4] Y. Alexahin, "Progress on the symplectic 3D Space Charge Kick Development", $2^{\text {nd }}$ CERN SC Collaboration Meeting, 2018.

[5] Y. Alexahin, "Adaptive Space Charge Calculations in MADX-SC”, NAPAC16-THPOA16, 2016.

[6] Y. Alexahin, "Computing Eigen-Emittances from Tracking Data", NAPAC16-THPOA17, 2016.

[7] R. L. Gluckstern, Phys. Rev. Lett. 73, 1247 (1994).

[8] G. Ripken, "Investigations On Disturbances In The Storage Ring Caused By The Detector Field And Their Corrections (In German)", DESY R1-70/5, June 1970.

[9] G. Ripken et al, “6D Beam-Beam Kick including Coupled Motion”, PRST-AB, Volume 3, Number 12, (2001).

[10] C. Iselin, "The MAD Program (Methodical Accelerator Design), Version 8.13, Physical Methods Manual" CERN/SL/92 (1992) (AP), chapter 8.5 therein.

[11] V. Kapin and F. Schmidt, "Frozen space charge model in MAD-X with adaptive intensity and sigma calculation", Workshop "Space Charge 2013", CERN, April, 2013; V. Kapin and F. Schmidt, "MADX-SC Flag Description", CERN-ACC-NOTE-2013-0036.

[12] N. D'Imperio, C. Montag, K. Yu, V. Kapin, E. McIntosh, H. Renshall and F. Schmidt, "Experience with OpenMP for MADX-SC", CERN-ACC-2014-0075, BNL C-A/AP/515.

[13] M. Titze, "Approach to combined-function magnets via symplectic slicing", PRST-AB, Volume 3, Issue 5, (2016).

[14] É. Forest, "Beam Dynamics: A New Attitude and Framework", Harwood Academic, pp.168-171 (1998).

[15] private communication M. Titze.

[16] private communication A. Valishev.

[17] Courtesy A. Huschauer.

[18] G. Franchetti, S. Gilardoni, A. Huschauer, F. Schmidt and R. Wasef, "Space charge effects on the third order coupled resonance", PRST-AB, Volume 20, Issue 8, (2017).

[19] H. Burkhardt, G. Rumolo, and F. Zimmermann, Proceedings of the Twentieth Particle Accelerator Conference, PAC2003, Portland, OR, USA, 2003, J. Chew and P. Lucas and S. Webber, pag. 30413043, IEEE, Piscataway, NJ, USA.

[20] H. Renshall, private communication. 
Report

\section{Appendix}

\section{A P \& Q Expansions}

Making notations $r=\frac{\sigma_{x}}{\sigma_{y}}, u=\frac{x}{\sigma_{x}}, v=\frac{y}{\sigma_{x}}$ we have:

$$
\begin{aligned}
P_{1} & =\pi\left(-1+r^{2}\right)\left[u^{2}-v^{2}\right] \\
P_{2} & =\frac{-3 \pi\left(-1+r^{2}\right)^{2}}{2}\left[u^{4}-6 u^{2} v^{2}+v^{4}\right] \\
P_{3} & =5 \pi\left(-1+r^{2}\right)^{3}\left[u^{6}-15 u^{4} v^{2}+15 u^{2} v^{4}-v^{6}\right] \\
P_{4} & =\frac{-105 \pi\left(-1+r^{2}\right)^{4}}{4}\left[u^{8}-28 u^{6} v^{2}+70 u^{4} v^{4}-28 u^{2} v^{6}+v^{8}\right] \\
P_{5} & =189 \pi\left(-1+r^{2}\right)^{5}\left[u^{10}-45 u^{8} v^{2}+210 u^{6} v^{4}-210 u^{4} v^{6}+45 u^{2} v^{8}-v^{10}\right] \\
P_{6} & =\frac{-3465 \pi\left(-1+r^{2}\right)^{6}}{2}\left[u^{12}-66 u^{10} v^{2}+495 u^{8} v^{4}-924 u^{6} v^{6}+495 u^{4} v^{8}-66 u^{2} v^{10}+v^{12}\right] \\
P_{7} & =19305 \pi\left(-1+r^{2}\right)^{7}\left[u^{14}-91 u^{12} v^{2}+1001 u^{10} v^{4}-3003 u^{8} v^{6}+3003 u^{6} v^{8}-1001 u^{4} v^{10}\right. \\
& \left.+91 u^{2} v^{12}-v^{14}\right] \\
P_{8} & =\frac{-2027025 \pi\left(-1+r^{2}\right)^{8}}{8}\left[u^{16}-120 u^{14} v^{2}+1820 u^{12} v^{4}-8008 u^{10} v^{6}+12870 u^{8} v^{8}-8008 u^{6} v^{10}\right. \\
& \left.+1820 u^{4} v^{12}-120 u^{2} v^{14}+v^{16}\right] \\
P_{9} & =3828825 \pi\left(-1+r^{2}\right)^{9}\left[u^{18}-153 u^{16} v^{2}+3060 u^{14} v^{4}-18564 u^{12} v^{6}+43758 u^{10} v^{8}-43758 u^{8} v^{10}\right. \\
& \left.+18564 u^{6} v^{12}-3060 u^{4} v^{14}+153 u^{2} v^{16}-v^{18}\right] \\
P_{10} & =\frac{-130945815 \pi\left(-1+r^{2}\right)^{10}}{2}\left[u^{20}-190 u^{18} v^{2}+4845 u^{16} v^{4}-38760 u^{14} v^{6}+125970 u^{12} v^{8}\right. \\
& \left.-184756 u^{10} v^{10}+125970 u^{8} v^{12}-38760 u^{6} v^{14}+4845 u^{4} v^{16}-190 u^{2} v^{18}+v^{20}\right]
\end{aligned}
$$

$$
\begin{aligned}
Q_{1} & =2 \pi\left(-1+r^{2}\right) u\left[-u^{2}+3 v^{2}\right] \\
Q_{2} & =6 \pi\left(-1+r^{2}\right)^{2} u\left[u^{4}-10 u^{2} v^{2}+5 v^{4}\right] \\
Q_{3} & =30 \pi\left(-1+r^{2}\right)^{3} u\left[-u^{6}+21 u^{4} v^{2}-35 u^{2} v^{4}+7 v^{6}\right] \\
Q_{4} & =210 \pi\left(-1+r^{2}\right)^{4} u\left[u^{8}-36 u^{6} v^{2}+126 u^{4} v^{4}-84 u^{2} v^{6}+9 v^{8}\right] \\
Q_{5} & =1890 \pi\left(-1+r^{2}\right)^{5} u\left[-u^{10}+55 u^{8} v^{2}-330 u^{6} v^{4}+462 u^{4} v^{6}-165 u^{2} v^{8}+11 v^{10}\right] \\
Q_{6} & =20790 \pi\left(-1+r^{2}\right)^{6} u\left[u^{12}-78 u^{10} v^{2}+715 u^{8} v^{4}-1716 u^{6} v^{6}+1287 u^{4} v^{8}-286 u^{2} v^{10}+13 v^{12}\right] \\
Q_{7} & =270270 \pi\left(-1+r^{2}\right)^{7} u\left[-u^{14}+105 u^{12} v^{2}-1365 u^{10} v^{4}+5005 u^{8} v^{6}-6435 u^{6} v^{8}+3003 u^{4} v^{10}\right. \\
& \left.-455 u^{2} v^{12}+15 v^{14}\right] \\
Q_{8} & =4054050 \pi\left(-1+r^{2}\right)^{8} u\left[u^{16}-136 u^{14} v^{2}+2380 u^{12} v^{4}-12376 u^{10} v^{6}+24310 u^{8} v^{8}-19448 u^{6} v^{10}\right. \\
& \left.+6188 u^{4} v^{12}-680 u^{2} v^{14}+17 v^{16}\right] \\
Q_{9} & =68918850 \pi\left(-1+r^{2}\right)^{9} u\left[-u^{18}+171 u^{16} v^{2}-3876 u^{14} v^{4}+27132 u^{12} v^{6}\right. \\
& \left.-75582 u^{10} v^{8}+92378 u^{8} v^{10}-50388 u^{6} v^{12}+11628 u^{4} v^{14}-969 u^{2} v^{16}+19 v^{18}\right] \\
Q_{10} & =1309458150 \pi\left(-1+r^{2}\right)^{10} u\left[u^{20}-210 u^{18} v^{2}+5985 u^{16} v^{4}-54264 u^{14} v^{6}+203490 u^{12} v^{8}\right. \\
& \left.-352716 u^{10} v^{10}+293930 u^{8} v^{12}-116280 u^{6} v^{14}+20349 u^{4} v^{16}-1330 u^{2} v^{18}+21 v^{20}\right] .
\end{aligned}
$$

